\title{
Preditores de Satisfação de Vida de Jovens em Situação de Vulnerabilidade no sul do Brasil
}

\author{
Débora Dalbosco Dell'Aglio•
}

Aline Cardoso Siqueira**

\section{Resumo}

Poucos estudos têm sido conduzidos sobre crianças e adolescentes que vivem em instituições, por terem sido afastados de suas famílias devido a maus-tratos. $\mathrm{O}$ objetivo deste estudo foi avaliar a satisfação de vida de jovens que estavam institucionalizados em abrigos de proteção $(n=155)$ comparados com jovens que viviam com suas famílias (n=142), com idade entre 7 e 16 anos. Os dados foram coletados através de entrevista estruturada, Escala de Satisfação Multidimensional de Vida, Inventário de Eventos Estressores e o Mapa dos Cinco Campos, para avaliar rede de apoio. O grupo institucionalizado apresentou muitas características de risco e médias mais altas nos escores em eventos estressores e rede de apoio social. As variáveis preditoras de satisfação de vida foram eventos estressores, rede de apoio social e conflitos familiares. Estes resultados apontam diferenças entre os grupos investigados e podem contribuir para a discussão de políticas públicas dirigidas ao bem-estar de jovens em situação de vulnerabilidade.

Palavras-chave: Institucionalização; Satisfação de vida; Vulnerabilidade.

\section{Abstract}

Few studies have been conducted on children and adolescents who were removed from their families and live in shelters because they were victims of maltreatment. The goal of this study was to assess the life satisfaction of children and adolescents living in shelters $(\mathrm{n}=155)$ compared with those living with their families $(\mathrm{n}=142)$, ages 7 to 16 years. Data were collected using a structured

- Doctora en Psicología. Universidade Federal do Rio Grande do Sul, Brasil. dalbosco@cpovo.net

-• Universidade Federal do Rio Grande do Sul, Brasil. 
interview, Multidimensional Life Satisfaction Scale, Stressful Events Inventory and the Five Field Map, to assess support network. The institutionalized group had many at-risk characteristics and significantly greater average scores on stressful life events and social support network. Factors that contributed to predicting life satisfaction were stressful life events, social support network, and family conflicts. The findings highlight differences between participants living in shelters compared to those living with their families. This article also discusses the policy implications of these findings for child welfare practice.

Key Words: Institutionalization; Life satisfaction; Vulnerability.

\section{Resumen}

Se han realizado pocos estudios sobre niños y adolescentes que viven en instituciones porque han sido alejados de sus familias debido a malos-tratos. El objetivo de este estudio fue evaluar la satisfacción de vida de jóvenes que han estado institucionalizados en casas de protección $(n=155)$ comparados con jóvenes que vivían con sus familias ( $\mathrm{n}=142)$, todos con edad entre 7 y 16 años. Los datos fueron recolectados a través de entrevista estructurada, Escala de Satisfacción Multidimensional de Vida, Inventario de Eventos Estresores y el Mapa de los Cinco Campos, para evaluar red de apoyo. El grupo institucionalizado presentó muchas características de riesgo y medias más altas en los escores en eventos estresores y red de apoyo social. Las variables de satisfacción de vida fueron eventos estresores, red de apoyo social y conflictos familiares. Estos resultados indican diferencias entre los grupos investigados y pueden contribuir para la discusión de políticas públicas dirigidas al bienestar de jóvenes en situación de vulnerabilidad.

Palabras Clave: Institucionalización; Satisfacción de vida; Vulnerabilidad. 


\section{Introdução}

No Brasil, milhares de crianças e adolescentes vivem em situação de pobreza e miserabilidade, com dificuldades de acesso a uma alimentação saudável, vestuário, educação, condições adequadas de moradia e habitação, entre outros aspectos (IBGE, 2002). Embora muitas vezes a pobreza não seja considerada como causa de problemas sociais, pesquisas têm demonstrado a sua associação com fatores de risco (Silva, 2004). Muitos dos jovens em situação de pobreza vivenciam eventos de vida negativos e estressores, que atuam como risco ao seu desenvolvimento. Estas situações de vida têm levado ao afastamento de crianças e adolescentes de suas famílias de origem, e a sua institucionalização em abrigos governamentais ou não-governamentais através da aplicação da medida de proteção, prevista pelo Estatuto da Criança e Adolescente (ECA, 1990), no Brasil. Na instituição de proteção, muitas crianças e adolescentes, ao mesmo tempo em que vivenciam sofrimento e sentimentos de abandono, advindos do afastamento familiar, também passam a ter acesso a condições de vida melhores e a uma estrutura organizada. Dessa forma, considerando a situação de crianças e adolescentes institucionalizados e daqueles que vivem com suas famílias em condições desfavorecidas de vida, o objetivo desse estudo foi investigar os preditores da satisfação de vida dessas crianças e adolescentes. Estudos que investiguem o bem-estar desses jovens em situação de vulnerabilidade social ainda são escassos na realidade brasileira, contudo, são importantes para melhor compreendê-los. Por tanto, serão verificadas as seguintes variáveis: eventos de vida estressores, satisfação multidimensional de vida, configuração familiar e rede de apoio social. Assim, torna-se necessário conceituar tais variáveis e revisar estudos sobre as mesmas.

\section{Bem-estar Subjetivo e Rede de Apoio Social e Afetivo}

O bem-estar subjetivo (BES), a partir de diferentes nomeações como felicidade, satisfação, estado de espírito, afeto positivo, e ainda, avaliação subjetiva da qualidade de vida, tem sido foco de estudos nas últimas décadas dentro da perspectiva da Psicologia Positiva (Giacomoni, 2002). O BES está relacionado ao que as pessoas pensam, avaliam e sentem sobre suas próprias vidas. Esta reflexão pode ser feita a partir de uma avaliação cognitiva ou em forma de afeto (emocional), dependendo de como serão considerados os aspectos relacionados ao sentimento de felicidade e satisfação. Se o julgamento é realizado a nível consciente, seja do todo ou de uma particularidade específica da vida, o indivíduo está avaliando de forma cognitiva; se esse julgamento leva em consideração os sentimentos, afetos, emoções prazerosas ou não, a avaliação é emocional (Diener, Suh, Lucas, \& Smith, 1999). 
Diversos autores apresentaram diferentes composições do construto "bemestar subjetivo”. Para Ryff e Keyes (1995), BES é composto por um componente afetivo, que corresponde ao afeto positivo e negativo (componentes emocionais); e um componente cognitivo, a satisfação de vida, envolvendo os aspectos racionais e intelectuais. Segundo Diener et al. (1999), BES é definido como uma ampla categoria de fenômenos, considerando as respostas emocionais das pessoas, domínios de satisfação e os julgamentos globais de satisfação de vida. A partir dessa definição, para estes autores, existem três componentes do bem-estar subjetivo: satisfação, afeto positivo e afeto negativo, sendo que estes componentes formam um fator global de variáveis inter-relacionadas.

A satisfação de vida, enquanto um componente da percepção de BES, está relacionada à avaliação cognitiva global, aos julgamentos que um indivíduo faz sobre sua própria vida. Para Lucas, Diener e Suh (1996), a avaliação pessoal inclui os aspectos reais da vida da pessoa, considerando os pontos positivos e negativos, e chegando a um julgamento de satisfação geral de vida. Este julgamento possui como características a estabilidade temporal e a independência do estado emocional da pessoa no momento da avaliação. Além da avaliação global, o indivíduo também pode emitir julgamentos de domínios específicos de sua vida, como trabalho, escola, amizades, amor, entre outros.

De acordo com Giacomoni (2002), modelos explicativos do BES têm sido desenhados com o objetivo de encontrar que fatores externos e internos influenciam a percepção de felicidade, não havendo um consenso na área. Entre as variáveis estudadas, encontram-se a idade, o nível educacional, a classe e a mobilidade social, a renda, o casamento, a etnia/raça, o desemprego, o lazer, a religiosidade, percepção e manejo de eventos estressores, entre outras variáveis, em amostras locais ou transculturais. Esta autora destaca a importância das características individuais e da personalidade para a percepção de felicidade, estando alguns indivíduos predispostos a interpretarem as situações e experiências de vida tanto de forma positiva quanto negativa. Assim, um indivíduo que apresente uma orientação pessoal e social positiva, perceberá de forma otimista eventos vivenciados adversos. Essa característica pessoal operará como fator de proteção ao seu desenvolvimento, conforme apontado por Masten e Garmezy (1985). Além das características individuais, como autonomia, auto-estima e orientação social positiva, estes autores destacam como fatores de proteção a presença de coesão familiar, ou seja, a disponibilidade de uma efetiva rede de apoio afetivo na família, e a disponibilidade de sistemas externos de apoio, interações sociais e afetivas mais amplas, que reforçam a capacidade do indivíduo de enfrentar as situações da vida. Estes três fatores de proteção são importantes para o desenvolvimento bem sucedido de crianças e adolescentes em situação de risco social e pessoal. A capacidade de estabelecer e manter vínculos afetivos dentro e fora da família, 
estabelecendo uma rede de apoio social e afetivo, constitui um fator de proteção para crianças e adolescentes.

Para Brito e Koller (1999), a rede de apoio social é definida como conjunto de sistemas e de pessoas significativas que compõem os elos de relacionamento recebidos e percebidos do indivíduo, enquanto que apoio social e afetivo está relacionado à percepção que a pessoa tem de seu mundo social, como se orienta nele, suas estratégias e competências para estabelecer vínculos, e com os recursos que esse lhe oferece, como proteção e força, frente a situações de risco que se apresentam. Para Sluzki (1997), a rede social pessoal pode ser definida como resultado da soma de todas as relações que um indivíduo percebe como significativas.

Segundo Garmezy e Masten (1994), a rede de apoio contribui para o aumento da competência individual, que reforça a auto-imagem e a auto-eficácia necessárias para alcançar um objetivo.

A rede de apoio social está associada à saúde e ao bem-estar dos indivíduos (Samuelsson, Thernlund, \& Ringström, 1996; Sluzki, 1997), sendo um fator fundamental para o processo de adaptação a situações de estresse e de suscetibilidade a distúrbios físicos e emocionais (Masten \& Garmezy, 1985; Rutter, 1987). O efeito protetivo que o apoio social oferece está relacionado ao desenvolvimento da capacidade de enfrentamento das adversidades, promovendo processos de resiliência, desenvolvimento adaptativo e superação (Brito \& Koller, 1999; Garmezy \& Masten, 1994; Rutter, 1987). Todas as relações que o indivíduo estabelece com as outras pessoas, advindas dos diversos micros sistemas nos quais transita, como família, amigos, escola, abrigo, entre outros, podem assumir o papel de fornecer apoio.

Para as crianças e adolescentes que vivem em instituições de abrigo, as pessoas com as quais esses convivem passam a integrar as suas redes de apoio social e afetivo, fazendo do abrigo o seu ambiente principal (Siqueira, Betts, \& Dell'Aglio, 2006; Yunes, Miranda, \& Cuello, 2004). É no abrigo que realizam um grande número de atividades, desempenham papéis e funções e interagem, abrindo espaço para o desenvolvimento de relações recíprocas, de equilíbrio de poder e de afeto estável (Bronfenbrenner, 1979/1996). Um estudo com adolescentes institucionalizados indicou uma média de 36 contatos na rede de apoio, sendo que a instituição apresentou maior média de contatos satisfatórios em comparação à família, e as meninas apontaram mais satisfação nas relações vivenciadas no abrigo do que os meninos, sendo estas diferenças significativas (Siqueira, Betts, \& Dell’Aglio, 2006). Assim, por mais que os motivos de entrada estejam associados a conflitos e sofrimento, as instituições de abrigo podem proporcionar um espaço seguro e protetivo, possibilitando acolhimento e relações satisfatórias, especialmente para as meninas (Siqueira, Betts, \& Dell’Aglio, 2006). 


\section{Eventos de vida e institucionalização}

Os eventos de vida podem ser influentes na percepção de bem-estar. São conceituados como sendo acontecimentos importantes vivenciados ao longo da história de vida, que podem ser positivos ou negativos. Os eventos positivos são aquelas situações que estão diretamente associadas à felicidade, alegria e bemestar. Os eventos negativos, ou estressores, em contrapartida, estão relacionados a situações de estresse, tristeza ou tensão, que desafiarão as condições internas do indivíduo. A percepção e o impacto dos eventos positivos e negativos dependem da avaliação subjetiva de cada indivíduo, que estará norteada pelas suas características pessoais, como autonomia, experiência prévia, além das características do seu meio social, como disponibilidade de apoio, de laços afetivos, entre outros (Dell'Aglio \& Hutz, 2000).

A vivência e o impacto de eventos negativos têm sido estudados pela comunidade científica. Esses eventos são considerados ameaçadores ao desenvolvimento saudável de crianças e adolescentes, pelo potencial prejuízo inerente, à medida que desafiam as condições internas de reação e adaptação. Para Lazarus e Folkman (1984), estresse pode ser entendido como um desequilíbrio entre as percepções do indivíduo quanto à demanda da situação e sua capacidade de manejá-las. A reação de estresse é um resultado da interação entre um agente causal, externo ou interno, uma avaliação cognitiva que diferencia entre modalidades do estressor (dano, ameaça e desafio) e os processos de coping utilizados para lidar com os estressores (Lazarus, 1993). Além disso, esta reação dependerá da avaliação subjetiva realizada pelo indivíduo, mediada pela cognição, fazendo com que respostas a uma mesma situação estressora sejam diferentes em diferentes indivíduos (Lazarus, 1993). A avaliação cognitiva é um processo que apresenta uma variabilidade no decorrer do tempo, sendo de difícil mensuração em certos casos (Grant, Compas, Stuhlmacher, Thurm, \& Halpert, 2003).

Pesquisas com crianças e adolescentes têm sido desenvolvidas, com o objetivo de constatar quais são os eventos estressores mais freqüentes e de maior impacto, e se existe especificidade quanto à idade e sexo ou diferença quanto ao contexto de origem. No Brasil, há estudos que focalizaram a percepção de eventos positivos e negativos na infância e adolescência (Dell’Aglio \& Hutz, 2000; Giacomoni, 2002; Kristensen, Leon, D’Incao, \& Dell’Aglio, 2004), e ainda, para a promoção de processos de resiliência (Pesce, Assis, \& Santos, 2004). Em geral, estes estudos têm apontado como eventos positivos mais freqüentes os eventos relacionados à família, lazer, ganhar presentes, brincar e ir à escola, enquanto que entre os negativos se destacam eventos relacionados a problemas de saúde, morte, violência, desentendimento familiar, desentendimento com pares, privação material ou problemas na escola. 
O estudo de Kristensen et al. (2004) investigou a freqüência dos eventos estressores de adolescentes. Qualquer situação vivida pelo indivíduo pode ser um evento estressor, desde mudança de escola ou cidade, férias, novo casamento dos pais, até eventos negativos, como violência doméstica. A avaliação subjetiva do indivíduo sobre os eventos estressores vivenciados depende da sua percepção, interpretação e sentidos atribuídos ao evento estressor, e é essa avaliação que o classificará ou não como condição de estresse (Pesce, Assis, \& Santos, 2004). Assim, um mesmo evento pode ser percebido como estressor por uma pessoa e benéfico por outra.

Os eventos de vida estressores podem ser considerados fatores de risco ao desenvolvimento humano, podendo levar ao surgimento de processos psicopatológicos relacionados à vulnerabilidade do indivíduo, entendida como uma predisposição para o desenvolvimento de disfunções psicológicas ou de respostas pouco adequadas à situação (Zimmerman \& Arunkumar, 1994). Os fatores de risco são definidos como condições ou variáveis que estão associadas a uma alta possibilidade de ocorrência de resultados negativos ou não desejáveis (Jessor, Van Den Boss, Vanderryn, Costa, \& Turbin, 1995), e os fatores de proteção, pelo contrário, são mecanismos ou processos que reduzem o impacto do risco e exercem efeitos positivos na saúde mental do indivíduo. Entretanto, deve-se compreender o conceito de fatores de proteção enfatizando-se uma abordagem de processos, através dos quais diferentes fatores interagem entre si e alteram a trajetória da pessoa, podendo produzir uma experiência estressora ou protetora em seus efeitos (Rutter, 1985, 1987).

Mesmo sendo o abrigamento uma medida de proteção, a institucionalização pode ou não ser vivenciada como um risco para o desenvolvimento. Esta condição dependerá dos mecanismos através dos quais os processos de risco operarão seus efeitos negativos sobre os abrigados (Yunes, Miranda, \& Cuello, 2004). Aspectos como acolhimento inadequado no momento do abrigamento, hostilidade entre crianças e monitores, práticas educativas coercitivas, rotatividade de funcionários e a não disponibilidade de investimento emocional podem ser considerados fatores de risco na institucionalização. Em contrapartida, inúmeros fatores de proteção podem operar neste momento, tais como acolhimento no momento do abrigamento, compreensão e respeito às histórias individuais de cada um, vinculação afetiva entre os abrigados e entre eles e os funcionários/monitores, sentimento de proteção, entre outros fatores, favorecendo o estabelecimento de novos relacionamentos e possibilitando o contato com uma estrutura organizada (Gulassa, 2006; Siqueira \& Dell’Aglio, 2007).

O panorama dos abrigos brasileiros tem se modificado nos últimos anos, após a implementação do ECA (1990), tendo em vista que antes as instituições de abrigo caracterizavam-se por grandes espaços físicos, nos quais muitas crianças e 
adolescentes viviam em regime coletivo, com horários rigidamente determinados para toda atividade e sob um atendimento padronizado, agrupados por sexo e idade (Cabral, 2002; Guirado, 1986). O principal objetivo do atendimento nessas instituições era fornecer condições básicas mínimas, não havendo uma preocupação com a individualidade e a conservação de laços familiares. Estas instituições, conhecidas como instituições totais, foram estudadas por inúmeros autores que, na grande maioria, destacavam os efeitos prejudiciais ocasionados pelo atendimento nas instituições (Bowlby, 1973/1998; Cabral, 2002; Guirado, 1986; Marin, 1999; Saraiva, 2002; Silva, 1997).

No Brasil, o Estatuto da Criança e do Adolescente (1990) destaca a convivência familiar e comunitária como direito fundamental, estabelecendo como prioridade a desinstitucionalização do atendimento à infância e à juventude em situação de risco e a reordenação das instituições existentes. Assim, estes abrigos devem assumir caráter residencial, oferecendo atendimento personalizado, em pequenas unidades e grupos reduzidos, entre outras determinações. Esse novo panorama preconizado pelo ECA (1990) tem possibilitado o entendimento de que as instituições de abrigo podem ser um contexto de desenvolvimento, proporcionando o provimento material e um ambiente seguro, além de possibilitar relações afetivas duradouras e estáveis.

Dessa forma, este estudo investigou os preditores da satisfação de vida em crianças e adolescentes institucionalizados e não-institucionalizados, advindos de famílias pobres, da região sul do Brasil, considerando a vivência de eventos estressores, a rede de apoio e características da família, entre outros aspectos.

\section{Método}

\section{Participantes}

Participaram desse estudo 297 crianças e adolescentes, sendo 155 jovens que viviam em abrigos governamentais e não-governamentais (institucionalizados) e 142 jovens que viviam com suas famílias (não-institucionalizados), de nível socioeconômico desfavorecido. Os participantes tinham de sete a 16 anos ( $M=11,21 ; D P=2,13$ ), sendo $52,2 \%$ do sexo masculino e $47,8 \%$, do sexo feminino. Os participantes do grupo não-institucionalizado foram selecionados em duas escolas públicas localizadas em regiões pobres da cidade de Porto Alegre/RS, Brasil. Os participantes do grupo de jovens institucionalizados foram selecionados em nove abrigos governamentais e não-governamentais, tendo uma média de 35,3 meses de tempo de abrigamento $(D P=35.14)$. 


\section{Instrumentos}

Foram utilizados os seguintes instrumentos:

1. Entrevista estruturada: A entrevista objetivou coletar dados sociodemográficos dos participantes, tais como gênero, idade, série e estrutura familiar.

2. Escala Multidimensional de Satisfação de Vida (EMSV, Giacomoni, 2002; Giacomoni \& Hutz, 2008): com 50 itens que avaliam seis dimensões da satisfação de vida: self, self-comparado, família, não-violência, amizade e escola. A EMSV é uma escala Likert de cinco pontos, variando de "nenhum pouco" (1) a "muitíssimo" (5). Esta escala apresentou consistência interna adequada (Alpha de Cronbach=0,93), e para este estudo, a média total da escala foi utilizada.

3. Inventário de Eventos Estressores na Infância e Adolescência (IEEIA; adaptado de Kristensen, et al., 2004): composto por 60 itens que investigam a ocorrência de eventos estressores de vida. Apresentou consistência interna adequada (Alpha de Cronbach $=0,90$ ).

4. Mapa dos Cinco Campos (Samuelsson, Thernlund, \& Ringström, 1996, versão adaptada por Siqueira et al., 2006): é um instrumento que avalia estrutura (quantidade de relações) e função (qualidade das relações) da rede, a partir de cinco campos: Família, Abrigo, Escola, Amigos/Parentes e Contatos Formais, representados de forma lúdica ao participante. A análise utilizou a média total de contatos, que foi derivada do número total de pessoas na rede de apoio social dos participantes e o número total de conflitos mencionados na família.

\section{Procedimentos e Considerações Éticas}

O projeto de pesquisa foi aprovado pelo Comitê de Ética em Pesquisa da Universidade Federal do Rio Grande do Sul e pelas direções das escolas e dos abrigos participantes. Foi obtido o Termo de Consentimento Livre esclarecido junto aos responsáveis pelos jovens e de todos os participantes maiores de 12 anos. A equipe de pesquisa realizou treinamento teórico, metodológico e ético para a coleta junto a crianças e adolescentes em situação de vulnerabilidade antes de iniciar a coleta de dados. Os jovens foram convidados a participar do estudo, sendo que a coleta foi realizada na escola, com o grupo não-institucionalizado, e nas instituições de abrigo, com o grupo institucionalizado, de forma individual.

Os dados coletados foram digitados em planilha eletrônica e analisados através do Programa SPSS.13. Para a descrição das características sociodemográficas dos participantes de ambos os grupos, foram utilizados os testes Qui-quadrado e Teste $t$ de Student. Uma regressão logística foi realizada para verificar os 
preditores da satisfação de vida, considerando as seguintes variáveis: eventos de vida estressores, rede de apoio social, configuração familiar e conflito na família. A variável satisfação de vida foi modificada de contínua para categórica, assim médias que variavam de zero a 3,9 foram transformadas em "0" (ausência de satisfação de vida), e médias que variaram de 3,91 a 5 foram transformadas em “1” (presença de satisfação de vida).

\section{Resultados}

A Tabela 1 apresenta as principais características sociodemográficas da amostra. Os grupos apresentaram semelhanças quanto ao sexo e idade, mas foram encontradas diferenças em outras variáveis. Houve diferença estatística na repetência escolar, sendo que o grupo institucionalizado apresentou média maior que o grupo nãoinstitucionalizado $\left(t_{(288,3)}=3,51 ; p=0,001\right)$. A análise indicou uma média de 3,9 irmãos nas famílias, sendo que o grupo de jovens institucionalizados apresentou média maior de irmãos do que o grupo não-institucionalizado $\left(t_{(288)}=3,3 ; p=0,001\right)$. Em 35,6\% dos casos, os pais moravam juntos. Contudo, a percentagem de pais que viviam juntos era significativamente maior $\left(X^{2}=45,08 ; d f=2 ; p<0,001\right)$ no grupo não-institucionalizado (54,3\%) do que no institucionalizado (17,1\%).

\section{Tabela 1 | Características sociodemográficas dos participantes}

\begin{tabular}{lccc}
\hline & Grupo institucionalizado & Grupo não-instituc. & $\boldsymbol{p}$ \\
\hline Sexo (\%) Masculino & 51 & 53,5 & 0,66 \\
\multicolumn{1}{c}{ Feminino } & 49 & 46,5 & 0,66 \\
\hline Idade (Média $\pm D P$ ) & $11,14 \pm 2,03$ & $11,27 \pm 2,24$ & 0,59 \\
\hline Repetência escolar & $1,2 \pm 1,05$ & $0,81 \pm 0,84$ & 0,001 \\
(Média anos $\pm D P$ ) & & & \\
\hline Irmãos (Média $\pm D P$ ) & $4,31 \pm 2,45$ & $3,4 \pm 2,24$ & 0,001 \\
\hline Pais que viviam juntos (\%) & 17,1 & 54,3 & $<0,001$ \\
\hline
\end{tabular}

A Tabela 2 apresenta os dados da satisfação multidimensional de vida, eventos de vida estressores, e rede de apoio social dos jovens institucionalizados e nãoinstitucionalizados. Para identificar a relação entre as variáveis nos grupos, foi utilizado Teste $t$ de Student. Foram constatadas diferenças estatisticamente significativas entre os grupos quanto à vivência de eventos estressores $\left(t_{(295)}=7,9\right.$; $p<0,001)$ e rede de apoio social $\left(t_{(295)}=9,9 ; p<0,001\right)$, sendo que o grupo de jovens institucionalizados apresentou maior média dessas variáveis. 
Tabela 2 | Média ( \pm desvio-padrão) da satisfação de vida, eventos de vida estressores e rede de apoio social por grupos

\begin{tabular}{lcccc}
\hline & $\begin{array}{c}\text { Grupo } \\
\text { institucionalizado }\end{array}$ & $\begin{array}{c}\text { Grupo não- } \\
\text { institucionalizado }\end{array}$ & Total & $p$ \\
\hline Satisfação de vida & $3,84 \pm 0,51$ & $3,94 \pm 0,48$ & $3,89 \pm 0,5$ & 0,085 \\
\hline Eventos estressores & $26,57 \pm 7,82$ & $18,88 \pm 8,96$ & $22,88 \pm 9,2$ & $<0,001$ \\
\hline Rede de apoio & $31,71 \pm 14,37$ & $18,45 \pm 8,1$ & $25,19 \pm 13,87$ & $<0,001$ \\
\hline
\end{tabular}

Os resultados da análise de regressão logística estão apresentados na Tabela 3. A análise indicou que eventos de vida estressores, rede de apoio social e presença de conflitos na família são os preditores da satisfação de vida para os jovens participantes do estudo.

Tabela 3 | Preditores da satisfação de vida $(\mathbf{n}=\mathbf{2 7 0})$

\begin{tabular}{lccccc}
\hline & & & & \multicolumn{2}{c}{$95 \%$ C. I. for EXP(B) } \\
\cline { 5 - 7 } Variáveis & B & SE B & Odds Ratio & Lower & Upper \\
\hline Eventos de vida estressores & $-0,05^{\star \star}$ & 10,31 & 0,95 & 0,92 & 0,98 \\
\hline Rede de apoio social & $0,32^{\star *}$ & 8,27 & 1,03 & 1,01 & 1,05 \\
\hline Conflitos na família & $-0,16^{*}$ & 4,72 & 0,85 & 0,73 & 0,98 \\
\hline Pais que viviam juntos & 0,22 & 0,97 & 1,25 & 0,8 & 1,95 \\
\hline Modelo $X^{2}$ & $23,75^{\star * *}$ \\
\hline Nagelkerke $R^{2}$ & 0,11 \\
\hline
\end{tabular}

Notas: ${ }^{*} p<.04 \quad{ }^{* *} p<.01 \quad{ }^{* * *} p<.001$

Os resultados indicaram que menor vivência de eventos estressores e de conflitos na família, e maior rede de apoio social estão associados à percepção de satisfação de vida. Para cada vivência adicional de evento estressor, as chances de apresentar satisfação de vida diminuem em 5,2\%. No que se refere à rede de apoio social, para cada unidade acrescentada, as chances de apresentar satisfação de vida aumentam em 33\%. Já para a variável conflitos na família, para cada unidade adicional, as chances de apresentar satisfação de vida diminuem em 14.9\%. Possuir os pais morando juntos não contribuiu para a satisfação de vida. 


\section{Discussão}

Os resultados revelaram semelhanças e diferenças entre as crianças e adolescentes que vivem em instituições de abrigo e aquelas que vivem com suas famílias, nos grupos pesquisados no Brasil. Os participantes dos dois grupos apresentaram alta taxa de repetência escolar, uma média de cerca de quatro irmãos e seus pais não estavam mais vivendo juntos, na maioria dos casos. Baixo grau de escolaridade, família monoparental e numerosa são características freqüentes na população de nível socioeconômico desfavorecido no Brasil. De acordo com o último censo do Instituto Brasileiro de Geografia e Estatística conduzido no Brasil (IBGE, 2000), o número de famílias chefiadas por mulheres aumentou de 7,7 milhões em 1991 para 12,8 milhões em 2000. Mais do que 28\% dessas mulheres viviam com um recurso mensal de cerca de 100 reais. Esta situação indica que muitas famílias brasileiras têm enfrentado problemas financeiros e acabam, muitas vezes, não conseguindo atender às necessidades mínimas básicas de seus filhos.

No presente estudo, a situação parece ser ainda pior para as crianças e adolescentes institucionalizados, pois muitos deles apresentam repetência escolar, possuem maior média de irmãos e menor percentagem de pais vivendo juntos, destacando a situação de risco em que se encontram. A literatura também tem discutido os efeitos negativos da institucionalização no desenvolvimento de crianças (Dell'Aglio \& Hutz, 2004; Saraiva, 2002). Além disso, pesquisas têm demonstrado que o desenvolvimento escolar e cognitivo está fortemente associado às condições socioeconômicas da família (Dell’Aglio \& Hutz, 2004; Ferreira \& Maturano, 2002).

Os participantes institucionalizados apresentaram média de eventos de vida estressores significativamente maior do que a do grupo que morava com a família. Uma vez que são jovens retirados das famílias em decorrência da presença de maus-tratos, abandono, violência doméstica, entre outros fatores, é compreensível que eles tenham vivenciado um número maior de eventos estressores. Este resultado também indica que os jovens não-institucionalizados, apesar das condições de vida desfavorecidas, estavam mais protegidos de eventos estressores e que de alguma forma suas famílias estavam cumprindo seu papel de proteção.

Considerando a rede de apoio social, os achados do presente estudo são semelhantes aos de outros estudos conduzidos com adolescentes institucionalizados (Siqueira et al., 2006). Contudo, a média encontrada para toda a amostra foi maior do que a encontrada em estudos com meninas vítimas de abuso sexual (Mayer, 2002) e crianças em situação de vulnerabilidade social (Hoppe, 1998). Outros estudos têm indicado que crianças e adolescentes institucionalizados têm uma expressiva rede de apoio visto que, uma vez que eles foram afastados da 
família e regressaram em um diferente contexto de interação, eles têm a oportunidade de desenvolver novas relações e incrementar sua rede de apoio.

Com relação à satisfação de vida, foi constatado que não houve diferença estatística entre os grupos, embora a média do grupo não-institucionalizado tenha sido maior. Embora o afastamento da família e a vivência institucional possam gerar sofrimento, este afastamento também protege a criança de fatores de risco experenciados nas famílias, como situação de maus-tratos. Ademais, jovens que cresceram em famílias em situação de risco apresentam dificuldades emocionais e sociais, e, além disso, muitos deles têm enfrentado problemas comuns em famílias pobres, como desemprego e carência de alimento. Nas instituições, os jovens têm suas necessidades básicas supridas, com acesso à alimentação, roupas, brinquedos, computadores e internet, entre outros que, em geral, não possuem quando estão junto a suas famílias. Associado a isso, no abrigo, as crianças e adolescentes têm acesso à escola e a freqüentam com assiduidade, fazem novos amigos dentro e fora do abrigo, através dos quais, o apoio social e emocional pode ser disponibilizado (Siqueira et al., 2006). Estes aspectos descritos podem incrementar a percepção de satisfação de vida dos jovens institucionalizados. Estudos têm indicado que as crianças e adolescentes possuem uma percepção positiva do abrigo e dos educadores/funcionários dos abrigos (Chapman, Wall, \& Barth, 2004; Oliveira, 2006; Siqueira \& Dell’Aglio, 2007). Além disso, as instituições de abrigo no Brasil são compostas em geral por grupos de cerca de 15 crianças e adolescentes, assistidos por educadores, monitores, casais ou pais sociais, segundo determinações do Estatuto da Criança e do Adolescente (1990). Este modelo de instituição, que busca uma aproximação com o sistema familiar, busca oferecer ao jovem uma série de atividades familiares, contrasta com as antigas instituições de abrigo brasileiras (Siqueira, Morais, Dell'Aglio, \& Koller, in press), que atendiam crianças e adolescentes em sistemas coletivizados.

A análise indicou que menor número de eventos de vida estressores, maior rede de apoio social e menos conflito na família se constituem em variáveis preditoras da satisfação de vida. Este resultado reitera outras pesquisas sobre rede de apoio social, nos quais apontam que pessoas que possuem apoio de pessoas significativas durante situações de estresse estão menos propensas ao desenvolvimento de comportamentos desadaptativos e problemas emocionais (Brito \& Koller, 1999; Garmezy \& Masten, 1994). Assim, a rede de apoio mostrou-se relacionada significativamente à satisfação de vida destes jovens, confirmando estudos que associam esta variável à saúde e ao bem-estar dos indivíduos (Samuelsson et al., 1996; Sluzki, 1997). O resultado de que um menor número de eventos estressores e menos conflito familiar se constituem em preditores da satisfação de vida também confirma estudos, nesta área, que apontam a vivência de eventos estressores como fator de risco ao desenvolvimento. No estudo de Abaid (2008), 
os eventos estressores e problemas familiares foram apontados como preditores da depressão em crianças e adolescentes, indicando sua importância para o bem-estar ao longo do desenvolvimento.

Por outro lado, a separação dos pais não foi identificada como uma variável preditora da satisfação de vida. Este resultado pode indicar que outros aspectos familiares são mais importantes na percepção de satisfação de vida, como qualidade dos relacionamentos familiares, entre os pais e entre pais e filhos, do que a composição da família. De fato, existem estudos que demonstram que a separação/divórcio dos pais pode levar a transformações positivas na família (Asmussen \& Larson, 1991; Leve \& Fagot, 1997). Além disso, Wagner, Ribeiro, Arteche e Bornholdt (1999) salientaram que o tipo de configuração familiar não tem sido associado ao bem-estar dos filhos adolescentes, recaindo na qualidade do relacionamento entre os membros da família a maior ou menor possibilidade de bem-estar dos adolescentes.

\section{Considerações Finais}

Os resultados do presente estudo apresentaram dados relativos a jovens que vivem em situação de vulnerabilidade social no Brasil, com informações importantes sobre as semelhanças e diferenças entre as crianças e adolescentes que vivem em abrigos e que vivem com suas famílias em condições desfavorecidas de vida. Os dados destacam muitos fatores de risco nos grupos, tais como pobre desempenho acadêmico, vivência de eventos estressores e famílias monoparentais. Eles também sugerem que o quadro é ainda mais grave para as crianças e adolescentes institucionalizadas, que apresentaram maior taxa de fracasso escolar, maior média de irmãos e menor percentagem dos pais vivendo juntos.

Pode-se observar que os participantes de ambos grupos apresentam pobres condições de vida, deixando claro que estas questões necessitam ser consideradas nas políticas de assistência social. Por outro lado, foi também constatada uma maior rede de apoio social entre os jovens institucionalizados, apontando melhorias nas condições dos abrigos e no seu funcionamento, segundo a percepção dos jovens, e indicando a efetividade da proposta do ECA (1990). No entanto, algumas limitações podem ser encontradas nesse estudo, tais como o uso de amostras por conveniência e amostras limitadas ao sul do Brasil, não sendo possível uma generalização para outras regiões brasileiras. Dessa forma, para um panorama amplo e completo do contexto institucional no Brasil, seria necessário investigar instituições de todas as partes do país, observando peculiaridades regionais. 
Os resultados deste estudo trazem contribuições para a compreensão da satisfação de vida entre crianças e adolescentes, apontando para a importância das relações familiares, da rede de apoio social disponível a estes jovens e da prevenção de eventos estressores ao longo do desenvolvimento. Assim, destaca-se a necessidade de políticas públicas que visem o fortalecimento das famílias e da rede de apoio, de forma que se possa favorecer o desenvolvimento saudável e a satisfação de vida de jovens em situação de vulnerabilidade.

\section{Referências}

Abaid, J. L. W. (2008). Vivências adversas e depressão: Um estudo sobre crianças e adolescentes institucionalizados. Dissertação de Mestrado. Programa de Pós-Graduação em Psicologia. Universidade Federal do Rio Grande do Sul, Porto Alegre, Brasil.

Asmussen, L., \& Larson, R. (1991). The quality of family time among young adolescents in single-parent and married-parent families. Journal of Marriage and the Family, 53(4), 1021-1030.

Bowlby, J. (1998). Apego e perda: Separação, angústia e raiva. São Paulo: Martins Fontes. (Original publicado em 1973).

Brito, R., \& Koller, S. H. (1999). Desenvolvimento humano e redes de apoio social e afetivo. In A. Carvalho (Ed.), O mundo social da criança: Natureza e cultura em ação (pp. 115-129). São Paulo: Casa do Psicólogo.

Bronfenbrenner, U. (1996). A ecologia do desenvolvimento humano. Porto Alegre: Artes Médicas. (Original publicado em 1979).

Cabral, C. (2002). Mudança de paradigma: Um desafio. In Terra dos Homens (Ed.), Cuidar de quem cuida: Reintegração familiar de crianças $e$ adolescentes em situação de rua (pp. 13-16). Rio de Janeiro: Booklink Publicações.

Chapman, M., Wall, A., \& Barth, R. (2004). Children's voices: The perceptions of children in foster care. American Journal of Orthopsychiatry, 74(3), 293-304.

Dell'Aglio D. D., \& Hutz, C. (2000). Depressão e desempenho escolar em crianças e adolescentes institucionalizados. Psicologia: Reflexão e Crítica, 17(3), 341-350.

Diener, E., Suh, E., Lucas, R. E., \& Smith, H. L. (1999). Subjective well-being: Three decades of progress. Psychological Bulletin, 125(2), 276-302. 
Estatuto da Criança e do Adolescente (1990). Diário Oficial da União. Lei $n^{o}$ 8069, de 13 de julho de 1990. Brasília, DF, Brasil.

Ferreira, M. C. T., \& Maturano, E. M. (2002). Ambiente familiar e os problemas do comportamento apresentados por crianças com baixo desempenho escolar. Psicologia: Reflexão e Crítica, 15(1), 35-44.

Garmezy, N., \& Masten, A. (1994). Chronic adversities. In M. Rutter, E. Taylor, \& L. Herson (Eds.), Child and adolescent psychiatry (pp. 191-207). Oxford: Blackwell.

Giacomoni, C. (2002). Bem-estar subjetivo infantil: Conceito de felicidade e construção de instrumentos para avaliação. Tese de Doutorado. Programa de Pós-Graduação em Psicologia. Universidade Federal do Rio Grande do Sul. Porto Alegre, Brasil.

Giacomoni, C., \& Hutz, C. S. (2008). Escala multidimensional de satisfação de vida para crianças: Estudos de construção e validação. Estudos de Psicologia, 25(1), 23-35.

Grant, K., Compas, B., Stuhlmacher, F., Thurm, A., McMahon, S., \& Halpert, J. (2003). Stressors and child and adolescent psychopatology: Moving from markers to mechanisms of risk. Psychologycal Bulletin, 129, 447-466.

Guirado, M. (1986). Instituição e relações afetivas: O vínculo com o abandono. São Paulo: Summus.

Gulassa, M. L. C. (2006). A fala dos abrigos. In M. V. Baptista (Ed.), Abrigo: Comunidade de acolhida e socioeducação (pp. 53-61). São Paulo: Instituto Camargo Corrêa.

Hoppe, M. (1998). Redes de apoio social e afetivo de crianças em situação de risco. Dissertação de Mestrado. Programa de Pós-Graduação em Psicologia. Universidade Federal do Rio Grande do Sul. Porto Alegre, Brasil.

Instituto Brasileiro de Geografia e Estatística. (2002). Censo Nacional do IBGE. Available at: http://www.ibge.gov.br/home/presidencia/noticias/ 26122003censofamiliashtml.shtm [Accessed 8 April 2008].

Jessor, R., Van Den Boss, J., Vanderryn, J., Costa, F., \& Turbin, M. (1995). Protective factors in adolescent problem behavior: Moderator effects and developmental change. Developmental Psychology, 31, 923-933.

Kristensen, C. H., Leon, J. S., D’Incao, D. B., \& Dell’Aglio, D. D. (2004). Análise da freqüência e do impacto de eventos estressores em uma amostra de adolescentes. Interação em Psicologia, 8(1), 45-55. 
Lazarus, R. S. (1993). From psychological stress to the emotions: A history of changing outlooks. Annual Review of Psychology, 44(1), 1-21.

Lazarus, R. S., \& Folkman, S. (1984). Stress, appraisal, and coping. New York: Springer.

Leve, L., \& Fagot, B. (1997). Gender-role socialization and discipline processes in one- and two-parent families. Sex Roles, 36(1), 1-21.

Lucas, R., Diener, E., \& Suh, E. (1996). Discriminant validity of well-being measures. Journal of Personality and Social Psychology, 71(3), 616-628.

Marin, I. S. K. (1999). FEBEM, família e identidade: O lugar do outro. São Paulo: Editora Escuta.

Masten, A., \& Garmezy, N. (1985). Risk, vulnerability and protective in developmental psychopathology. In B. Lahey \& A. Kazdin (Eds.), Advances in clinical child psychology (pp. 1-52). New York: Plenum Press.

Mayer, L. R. (2002). Rede de apoio social e afetivo e representação mental das relações de apego de meninas vítimas de violência doméstica. Tese de Doutorado. Universidade Federal do Rio Grande do Sul. Porto Alegre, Brasil.

Oliveira, R. C. (2006). A história começa a ser revelada: Panorama atual do abrigamento no Brasil. In M. V. Baptista (Ed.), Abrigo: Comunidade de acolhida e socioeducação (pp. 39-51). São Paulo: Instituto Camargo Corrêa.

Pesce, R., Assis, S., \& Santos, N. (2004). Risco e proteção: Uma busca de um equilíbrio promovedor de resiliência. Psicologia: Teoria e Pesquisa, 20, 135-143.

Rutter, M. (1985). Resilience in the face of adversity: Protective factors and resistance to psychiatric disorder. British Journal of Psychiatry, 147, 598-611.

Rutter, M. (1987). Psychosocial resilience and protective mechanisms. American Journal Orthopsychiatry, 57(1), 316-331.

Ryff, C. D. \& Keyes, C. L. M. (1995). The structure of psychological well-being revisited. Journal of Personality and Social Psychology, 69, 719-727.

Samuelsson, M., Thernlund, G., \& Ringström, J. (1996). Using the five field map to describe the social network of children: A methodological study. International Journal Behavioral Development, 19(2), 327-345.

Saraiva, J. E. M. (2002). Contextualizando a problemática. In Terra dos Homens (Ed.), Cuidar de quem cuida: Reintegração familiar de crianças e adolescentes em situação de rua (pp. 7-12). Rio de Janeiro: Booklink Publicações. 
Silva, E. R. (2004). O direito à convivência familiar e comunitária: Os abrigos para crianças e adolescentes no Brasil. Brasília: IPEA/CONANDA.

Silva, R. (1997). Os filhos do governo. São Paulo: Editora Ática.

Siqueira, A. C., \& Dell’Aglio, D. D. (2007). Retornando à família de origem: Fatores de risco e proteção no processo de reinserção familiar de uma adolescente institucionalizada. Revista Brasileira de Crescimento e Desenvolvimento Humano, 17(3), 134-146.

Siqueira, A. C., Betts, M. K., \& Dell’Aglio, D. D. (2006). Rede de apoio social e afetivo de adolescentes institucionalizados no Sul do Brasil. Interamerican Journal of Psychology, 40(2), 149-158.

Siqueira, A. C., Morais, N. A., Dell'Aglio, D. D., \& Koller, S. H. (in press). Experiência das casas lares: Uma alternativa possível para crianças e adolescentes em situação de rua. In N. A. Morais, L. Neiva-Silva \& S. H. Koller (Eds.), Endereço desconhecido: Crianças e adolescentes em situação de rua. Porto Alegre: Casa do Psicólogo.

Sluzki, C. (1997). A rede social na prática sistêmica: Alternativas terapêuticas. São Paulo: Casa do Psicólogo.

Wagner, A., Ribeiro, L. de S., Arteche, A. X., \& Bornholdt, E. A. (1999). Configuração familiar e o bem-estar psicológico dos adolescentes. Psicologia: Reflexão e Crítica, 12(1), 147-156.

Yunes, M. A., Miranda, A. T., \& Cuello, S. S. (2004). Um olhar ecológico para os riscos e as oportunidades de desenvolvimento de crianças e adolescentes institucionalizados. In S. H. Koller (Ed.), Ecologia do desenvolvimento humano: Pesquisa e intervenções no Brasil (pp. 197-218). São Paulo: Casa do Psicólogo. Zimmerman, M. A. \& Arunkumar, R. (1994). Resilience research: Implications for schools and policy. Social Policy Report, 8, 1-18. 